\title{
LA EDUCOMUNICACIÓN APLICADA EN TELEVISIONES LOCALES
}

\section{EDUCOMMUNICATION APPLIED TO LOCAL TELEVISION BROADCASTING}

José Israel Méndez Ojeda Universidad de Yucatán, México isra6996@hotmail.com

Sergio Luque Ortiz Universidad de Sevilla, España sluqueortiz@gmail.com

Concha Pérez Curiel Universidad de Sevilla, España cperez1@us.es

\section{Resumen}

Este artículo estudia la relación existente entre la televisión, la comunicación y la educación en valores. La investigación analiza la importancia de la educomunicación -o educación en los medios de comunicación-con una comparación entre dos experiencias educomunicativas reales en una comunidad local indígena del estado de Yucatán (México) y en un instituto de enseñanza secundaria obligatoria de Córdoba (España). Mediante la aplicación del método cualitativo de la encuesta se medirá la importancia que tiene el modelo educomunicativo en las aulas, con la finalidad de enseñar comunicación como un factor poderoso de cambio social y cultural. Los resultados muestran que la participación de las audiencias en el diseño y construcción de contenidos televisivos multiplica la eficacia en educación en valores y la formación del espíritu crítico, de las experiencias educomunicativas.

Palabras clave: Educomunicación, audiencia, educación, información, comunicación, cultura, aulas, estudiantes, televisión, medios de comunicación

\begin{abstract}
:
This article studies the relationship between television- and communicationbased education and the formation of values. The research analyzes the importance of educommunication, or education via the communication media, by comparing two real educommunicative experiences: one in a local indigenous community in the state of Yucatan (Mexico), and the other in a group of secondary education students in Cordoba (Spain). By applying the qualitative survey method, one will be able to measure the importance of the educommunicative model in classroom training centers, with the goal of teaching communication in the classroom as a powerful factor of social and cultural change. Results demonstrate that the participation of audiences in design and construction of television content multiplies the effectiveness of educommunication in values education and formation of a critical spirit.
\end{abstract}

Keywords: Educomunication, audience, education, information, communication, culture, classes, students, television, media. 


\section{Introducción}

Esta investigación analiza la importancia de la educomunicación en los centros educativos de enseñanzas medias y superiores, tanto a nivel nacional como internacional. Se ha realizado un estudio de la educomunicación entre estudiantes de México y España, teniendo como principal herramienta de análisis el desarrollo de un programa de televisión bajo parámetros educativos y la posterior medición de los resultados mediante el método encuesta.

La investigación se ha desarrollado paralelamente entre los miembros de la comu $\neg$ nidad indígena del municipio de Ucú, del Estado de Yucatán (México) y estudiantes de educación secundaria obligatoria del instituto Tirso de Molina, Córdoba (España). En ambos casos se ha implementado la importancia de la educomunicación como un modelo viable de educación en valores sociales, independientemente de la ubicación geográfica. Pero antes de explicar con más detalle en qué ha consistido la investigación, es necesario aportar datos sobre el origen y las características más importantes de la educomunicación. El término educomunicación no podría definirse con una sola palabra. Diferentes autores consideran que la educomunicación es un campo de estudio de dos disciplinas: la educación, entendida como alfabetización mediática, y la comunicación, como una extensión de la pedagogía del conocimiento. La comunicación y educación no pueden ni deben entenderse de manera separada. La eficacia del proceso comunicativo depende en buena parte de la educación-comunicación y de la comunicación-educación (De Oliveira, 2000; Valderrama, 2000; Martín-Barbero, 2001).

Para entender mejor qué es educación y qué es comunicación acudimos al diccionario de la RAE: 'educación' cómo acción o efecto de enseñar/ crianza y doctrina que se les da a los niños y a los jóvenes/ instrucción por medio de la acción docente, cortesía y urbanidad $\left(1^{\circ}, 2^{\circ}, 3^{\circ}\right.$ acepción de la palabra 'educación'). Según la RAE, la 'comunicación' es la acción o el efecto de comunicarse/ trato o correspondencia entre dos o más personas/ transmisión de señales mediante un código común entre emisor-receptor/ unión que se establece entre ciertas cosas/ papel escrito en el que se comunica algo oficialmente $\left(1^{\circ}, 2^{\circ}, 3^{\circ}, 4^{\circ}\right.$ y $5^{\circ}$ acepción de la palabra 'comunicación').

Esta investigación analiza la experiencia educomunicativa en dos escuelas situadas en México y en España. Para conocer mejor los resultados debemos entender cuáles han sido las aportaciones de los teóricos españoles en el ámbito de la educomunicación. Nuestro país es uno de los países europeos en los que más experiencias educomunicativas se han realizado. Así, diferentes académicos y expertos han sumado esfuerzos para continuar la lucha en pro de la educomunicación como un modelo necesario a implantar en las escuelas españolas. Investigadores como José Ignacio Aguaded, José Manuel Pérez Tornero y Jesús Martín Barbero, entre otros teóricos, consideran que las escuelas deberían modificar el modelo educativo reinante dando paso a una dinámica práctica, participativa, y sobre todo, real, haciendo de la educación un potente aliado del cambio social.

En este sentido, la educación debe reinventarse. El modelo educativo basado en la lectura del libro, de arriba hacia abajo, y de izquierda a derecha, lineal, haciendo en gran parte que el alumno repita cada una de las palabras que ha dicho el maestro, tiene que transformarse. Ya no es una voz del maestro que incita a hablar, a escribir, a pensar, a crear. Más allá de la buena voluntad del docente es una cuestión del modelo de comunicación en la educación. Hay una esquizofrenia entre el modelo de comunicación social, que es mucho más abierto, mucho más de red, mucho más complejo y el modelo de comunicación escolar que sigue siendo jerárquico (Martín 
Barrero, 2002). Según el argumento dado por Jesús Martín Barbero, el reto de la educomunicación implica una formación específica en el área por parte del cuerpo docente de los centros educativos.

Ignacio Aguaded, presidente del grupo Comunicar, docente de Educación y Comunicación de la Universidad de Huelva (España), es uno de los grandes expertos en educomunicación a nivel nacional. Para este teórico, la educomunicación es una apuesta de mañana necesaria para hoy, es decir, la presencia de los medios de comunicación a nivel social es especialmente visible entre las nuevas generaciones de niños. De estas complejas relaciones de los niños y jóvenes con los medios de comunicación, surge la necesidad de planificar y proyectar una educación para el conocimiento de estos nuevos lenguajes, en el contexto de una sociedad cada vez más mediatizada, e indagar sobre como la educación, en general, y la enseñanza, en particular, han de responder el papel central que los medios de comunicación tienen en la vida de los jóvenes. En ese sentido, es cada vez más necesaria una educación en los medios de comunicación, entendida como un ámbito de estudio la educación de la "competencia educativa" (Aguaded, 2012).

Frente a las visiones de Martín Barbero y Aguaged, destaca la particular visión del proceso educomunicativo esgrimida por José Manuel Pérez Tornero. Este autor ha participado en diferentes iniciativas en las que la educación y la comunicación han sido protagonistas absolutas. Según Pérez Tornero, son necesarias nuevas competencias comunicativas en los contextos mediáticos, además de dominar el proceso de nuevos procesos de medición que vayan del texto al hipertexto, del medio al hipermedia, sobre una concepción alternativa del lenguaje como la integración de múltiples lenguajes que, juntos, producen sentido y coherencia. El progreso de la tecnología de la comunicación debe tener una aplicación directa en los procesos educativos porque los métodos tradicionales de enseñanza, de lectura, de cálculo, serán sustituidos por métodos audiovisuales, con más nervio, con un ritmo distinto que conecte de verdad con la sociedad. El problema que se planteará en un futuro inmediato es qué generación de profesores estará preparada y dispuesta para asumir este cambio transversal en la educación (Pérez Tornero, 2012).

En el ámbito latinoamericano, destaca la aportación de grandes expertos en educomunicación como Paulo Freire, Antonio Pascuali o Mario Kaplún. La importancia que el docente tiene en las aulas es indiscutible. El profesional de la educación no sería nada sin la presencia del alumnado deseoso de aprender. Sin embargo, profesor no es el único que educa, sino que cuando educa también es educado, en diálogo continúo con el educando que al ser educado también educa. Los hombres y mujeres se educan en comunión, impulsados por el mundo en el cual viven y tomando parte de las relaciones con los instrumentos sociales (Freire, 1978).

La educación, como práctica de la libertad, precisa el sentido comunicativo de su método y la condición ineludible de su propuesta. En las relaciones humanas de gran dominio, la distancia social existente impide el diálogo entre hombres. El diálogo se da en las áreas abiertas, donde el hombre desarrolla su sentido de participación en la vida común, lo que implica la responsabilidad política y social del hombre, es una relación entre personas que nace de una matriz crítica [...]. El diálogo sirve para comunicar. Cuando los polos se ligan así, con amor, esperanza y fe, uno en el otro, se hacen críticos en la búsqueda de algo. Entonces se genera una relación de simpatía entre ambos. Solo ahí hay verdadera comunicación (Freire, 1997).

Otra aportación en el ámbito de la educomunicación es la ofrecida por Antonio Pascuali. En comunicación y educación para el cambio social interesa más el proceso 
que los propios productos en sí mismos. Esta fase debe promover la erradicación de fronteras y estimular la creación de contenidos desde la igualdad, el respeto y la multiplicidad. Además, la pertinencia cultural de las acciones que se realicen debe tener coherencia con las propias características culturales. Por último, han de evitarse las sobredimensiones en torno a las diferencias individuales (Pascuali, 1963).

Mario Kaplún, considerado como un revolucionario de la educomunicación por sus trabajos en Lationamérica, adoptó la perspectiva de comunicación-educación como un servicio para la comunidad. La reflexión de Kaplún explora la relación existente entre desarrollo-educación-comunicación. La suma de estos factores ayuda a comprender el rol de emisor y receptor, tomando como punto de partida el axioma de que no es posible separar comunicación y cognición. Únicamente cuando el sujeto educando pueda expresar una idea de modo que los otros puedan comprenderla, es cuando él mismo la aprende e interioriza realmente (Kaplún: 1993). Para este autor, el lenguaje es instrumento de pensamiento. Sólo aquel contenido que hemos tenido ocasión de comunicar es susceptible de ser asimilado convirtiéndose en vehículo del cambio social (Kaplún: 1998). Según sus aportaciones, comunicación y educación son parte de un todo indivisible relacionado con la política, la economía y el cambio. Este autor considera que cada tipo de educación tiene una determinada práctica de la comunicación distinguiendo tres tipos (Kaplún: 1985):

- La educación que enfatiza en los contenidos o pedagogía transmisora.

- La educación que pone el foco de atención en los efectos o persuasivo conductivista. Se caracteriza por el intento de moldear la conducta de los educandos según cánones establecidos por el educador.

- Por último, la educación que subraya el proceso comunicativo en sí mismo o educación problematizadora.

Una de las grandes aportaciones de Kaplún (1998) fue la creación del término prealimentación. Este concepto, impuesto durante los años 70 y 80 , se define como: "la búsqueda inicial que hacemos entre los destinatarios de nuestros medios de comunicación para que nuestros mensajes los representen y reflejen" (Kaplún, 1998, 78-79). El concepto de prealimentación implica que el comunicador debe centrar su labor en el respeto desarrollando un tipo de conciencia profunda sobre lo que otros desean.

\section{La educomunicación en el tiempo}

Las primeras experiencias educomunicativas en centros educativos fueron desarrolladas por Célestin Freinet y por Frank Raymond Leavis, en torno a las décadas de 1920 y 1930. Célestin Freinet, considerado un pionero y uno de los padres de la educomunicación, investigó sobre los efectos que tenía la introducción de los medios de comunicación en las aulas. Así, Freinet, impulsó la creación de una revista escolar en un centro educativo francés, con el objetivo de que los estudiantes escribieran sobre las noticias que les afectaban y sobre acontecimientos que formaban su día a día. El interés de los alumnos en esta iniciativa fue creciente. Los estudiantes consideraban la revista como un medio para expresar sus inquietudes personales, culturales, sociales, además de ser un canal de expresión libre que fomentaba la comunicación entre los compañeros, pero también entre los profesores de la escuela primaria.

Len Masterman -consultor de la UNESCO- considera que los primeros pasos en la educomunicación se dieron en Gran Bretaña con las aportaciones de Leavis y 
Thompson (1933). Entre los años 40 y 50, la educomunicación vivió un desarrollo sin precedentes extendiéndose en los años 70 mediante programas de integración escolar impulsados por la UNESCO. Este organismo internacional fomentó la implantación de la educomunicación en escuelas de América del Sur constatándose en las obras de importantes autores latinoamericanos como Paulo Freire, Mario Kaplún o Francisco Gutiérrez, entre otros. Uno de los grandes hitos en la historia reciente de la educomunicación ha sido la creación de la Comisión Internacional para el Estudio de los problemas de Comunicación bajo la presidencia del Premio Nobel de la Paz, Sean MacBride, autor del famoso Informe McBride, publicado en 1980, cuyo principal objetivo es claro: hacia un nuevo orden mundial de la comunicación, más justo y más eficiente. Durante los años 70 y 80 , la educación aplicada a la comunicación se extiende en diferentes partes del mundo con desiguales grados de seguimiento. En este contexto, los modelos clásicos pierden importancia frente a nuevas alternativas receptoras de las necesidades culturales, sociales, económicas y emocionales.

A pesar del desarrollo que la educomunicación ha experimentado en México, Perú, Brasil, Gran Bretaña y España, aún queda mucho por hacer. Existen varios obstáculos que dificultan la plena aplicación de la educomunicación como método de enseñanza. La falta de conocimiento en la materia se une a la ausencia de docentes especializados en impartir contenidos específicos en educación y comunicación, no como dos disciplinas aíslas entre sí, tal como expresamos en párrafos anteriores, sino como un nexo de unión para el cambio de comportamientos sociales en la búsqueda de la democratización del conocimiento y la formación de ciudadanos para la democracia. Consideramos oportuno resaltar que esta investigación se ha desarrollado paralelamente en centros educativos de México y España. Ambos países tienen una dilatada experiencia en el desarrollo de experiencias educomunicativas, viéndose completadas por las aportaciones de algunos de los teóricos estudiados más arriba.

\section{Educomunicación a través de la televisión estudiantil}

La investigación aborda la experiencia educomunicativa y dos escuelas con el objetivo de medir resultados y comparar la importancia del modelo educomunicativo en centros docentes.

El objeto de estudio es determinar, conocer, estudiar y analizar cómo se realiza un proceso educomunicativo, basado en el desarrollo de una televisión estudiantil, entre el alumnado de una comunidad rural mexicana y estudiantes de un instituto de Córdoba, y medición de resultados a través de la encuesta.

Las hipótesis manejadas son: 1. La experiencia educomunicativa sirve para que los estudiantes aprendan valores que posteriormente pondrán en práctica en cursos superiores o en el futuro profesional. 2. La educomunicación es un modelo de aprendizaje que garantiza el pluralismo, la libertad y la educación en valores frente a la distorsión de información del discurso televisivo actual imperante en cadenas de televisión.

Respecto a la metodología, el procedimiento seguido comprende dos fases que a continuación se muestran con más detalle.

Fase 1: Desarrollo de la acción educomunicativa. El ejercicio educomunicativo ha tenido lugar entre estudiantes procedentes de una comunidad indígena de México y estudiantes de un centro de educación secundaria de Córdoba. La experiencia 
educomunicativa ha consistido en la creación de un programa de televisión entre los estudiantes, con el objetivo de dotar de espíritu crítico a los alumnos. De todos los medios de comunicación existentes, los estudiantes eligieron la televisión. Este medio de comunicación es el más cercano a los chicos que participaron en la investigación. Todos tienen una en casa. La variedad del contenido hace de la televisión un género muy completo y vivo. A partir de esto, se generó un programa denominado "Televisión Educativa", tanto en México como en España.

La comunidad rural de Ucú en México tiene un perfil particular. La escuela primaria, compuesta por 150 alumnos (de entre 5 y 16 años) y 6 profesores, realiza diferentes acciones para incentivar el conocimiento, la formación de espíritu crítico y la creatividad entre el alumnado. Sin embargo, los alumnos seleccionados sabían muy poco sobre técnicas de comunicación, géneros periodísticos o formas de interrelación. Para mejor estas deficiencias, se planteó el proyecto educomunicativo a través del magazine (género televisivo caracterizado por la variedad de contenidos: entrevistas, debates, crónicas, noticiero).

En el caso del grupo de estudiantes procedentes del instituto de educación secundaria Tirso de Molina de Córdoba, con edades comprendidas entre los 12 y 16 años, cabe destacar su elección sin diferencias por el uso de la televisión, frente a la radio o la prensa. Se crearon espacios de colaboración y aprendizaje en el que los estudiantes, los profesores e incluso los padres y las madres participaron activamente en la selección de las noticias, búsqueda de contenidos, elaboración del magazine y roles de cada uno de los estudiantes en este aventura televisiva. El contexto de aprendizaje creado ha permitido la implementación de un modelo novedoso de relaciones sociales, contacto y comunicación directa entre todos los implicados en el proceso educativo, al tiempo que dedica especial atención en aquellos niños que presentan unas necesidades educativas especiales, con peor nivel de conocimientos culturales y sociales o que proceden de familias con problemas internos.

En todo el proceso de elaboración del magazine, la vinculación del entorno con el poder educomunicativo estuvo presente. Los productos educomunicativos en la televisión libre brillan por su ausencia. De ahí la necesidad de crear un nuevo producto que sirva para marcar un antes y un después en las escuelas. Los niños eligieron los contenidos del programa de televisión, previo consenso con profesores de la comunidad de Ucú en México y con docentes del instituto de ESO Tirso de Molina en Córdoba. A continuación se muestran los diferentes bloques temáticos del programa:

- Un espacio dedicado a la actividad física y hábitos de vida saludable.

- Un espacio de higiene bucal y prevención de enfermedades dentales.

- Lectura de cuentos tradicionales y poemas.

- Juegos de entretenimiento para conocer otras culturas y formas de pensar.

Definidos los contenidos, los estudiantes seleccionados construyeron el proyecto periodístico aprendiendo a trabajar según rutinas periodísticas. El reparto de funciones contenía diferentes actividades como: redacción, edición, locución, montaje, grabación, programación, etcétera. Los alumnos han aprendido a formalizar el contenido de los medios de comunicación

Fase 2: Medición del proyecto educomunicativo. La segunda fase de la investigación concluye con la medición del proyecto educomunicativo. Una vez realizado el programa de televisión, entre estudiantes mexicanos y estudiantes españoles, se cuantifi- 
caron las impresiones, las opiniones y las experiencias con respecto al experimento planteado. El grupo de trabajo ha utilizado la técnica de la encuesta.

La encuesta es una técnica de obtención de información mediante un cuestionario previamente elaborado, a través del cual se obtienen opiniones o valoraciones de ciertos sujetos seleccionados, más conocidos como muestra, o grupo de estudio sobre un asunto determinado. En la encuesta, el encuestado lee previamente el cuestionario y responde sin la intervención de ninguna persona que pueda alterar las respuestas o condicionar sus opiniones ante las cuestiones planteadas. Otro de los aspectos más importantes de la encuesta es que tiene una estructura lógica, rígida y muy concreta, permaneciendo inalterable a lo largo de todo el proceso investigativo.

Esta técnica de trabajo se define como la aplicación o puesta en práctica de un procedimiento estandarizado para recibir información (oral o escrita) de una muestra amplia de sujetos. La muestra ha de ser representativa de la población de interés y la información recogida se limita a la delineada por las preguntas que componen el cuestionario precodificado, diseñado con anterioridad a tal efecto (Cea, 1999).

Mediante la encuesta pretendemos medir diferentes aspectos y parámetros conducentes a determinar la importancia del proceso educomunicativo entre los estudiantes seleccionados. Existen diferentes tipos de encuestas y de cuestiones. En el caso de esta investigación, el grupo de trabajo optó por encuestas de tipo descriptivo. Este tipo de formularios es adecuado para obtener información sobre un acontecimiento puntual y concreto como es el caso del experimento educomunicativo. Otro de los aspectos más importantes de la encuesta es el tipo de pregunta a formular. La investigación se ha desarrollado gracias a la participación de los estudiantes al responder a diferentes cuestiones con respuesta abierta dando mayor libertad a los entrevistados al responder a las preguntas de una forma más profunda y exhaustiva. Cada una de las preguntas tiene tres posibles opciones de respuesta.

Muestra. Para entender los diferentes aspectos que conforman la investigación es necesario tener más datos sobre la muestra sobre el que se dirige la acción de educomunicación.

1.Selección de 60 estudiantes con edades comprendidas entre los 12 y los 16 años, procedentes de la comunidad indígena del municipio de Ucú, integrada en el Estado de Yucatán, al Noroeste de la ciudad de Mérida (México).

2.Selección de 60 estudiantes con edades comprendidas entre los 12 y 16 años, procedentes del instituto de educación secundaria obligatoria Tirso de Molina en Córdoba, España.

3. Medición de la experiencia educomunicativa mediante encuestas entregadas a los estudiantes seleccionados (40 mexicanos y 40 españoles), con el objetivo de medir datos definitivos que certifican la idoneidad del método educomunicativo en las aulas nacionales e internacionales.

La muestra se ha seleccionado sobre 60 alumnos, frente a un total de 120 alumnos pertenecientes a los centros educativos analizados. El diseño de la muestra ha servido para conocer datos específicos sobre los intereses de los estudiantes en el método educomunicativo. Mediante la encuesta puede determinarse si la educomunicación puede convertirse en una práctica habitual en los centros educativos. Las variables que se han analizado tienen que ver con el interés que tienen los estudiantes en la educomunicación, como una plataforma para expresar sus problemas, necesidades o simplemente, cuestiones que no se atreven a preguntar a sus profesores y a los padres. Los encuestados han tenido que responder a las siguientes cuestiones: 
1. ¿Conocías el método de aprendizaje basado en la educomunicación?

\begin{tabular}{|l|}
\hline a) Sí \\
\hline b) No \\
\hline c) Ns/Nc \\
\hline 2. ¿Has aprendido más con el método educomunicativo o con el método tradicional? \\
\hline a) Con el método educomunicativo \\
\hline b) Con el método tradicional \\
\hline c) Con ninguno de los dos \\
\hline 3. ¿Cuál ha sido para ti el principal interés de esta actividad? \\
\hline a) Aprender desde el conocimiento práctico \\
\hline b) Adquirir un aprendizaje participativo \\
\hline c) Relacionarme con otros compañeros de clase \\
\hline 4. La educomunicacion es: \\
\hline a) Un modelo de educación basado en la enseñanza desde los medios de comunicación \\
\hline b) Un método de estudio práctico \\
\hline c) Una nueva dinámica de estudio teórico \\
\hline 5. ¿Qué has aprendido mediante el magazine televisivo? \\
\hline a) Trabajo en equipo \\
\hline b) Desarrollo de áreas personales \\
\hline c) Comportamiento grupal \\
\hline $\begin{array}{l}\text { 6.¿Qué diferencias observas entre el programa de televisión elaborado y el resto de contenidos en } \\
\text { la televisión? }\end{array}$ \\
\hline a) La calidad \\
\hline b) Un contenido acorde a mis necesidades \\
\hline c) La educación en valores \\
\hline
\end{tabular}

\section{Resultados}

\subsection{Resultados de estudiantes mexicanos}

A la primera pregunta planteada a los estudiantes de la comunidad indígena de Ucú, los estudiantes muestran un desconocimiento casi total de la educomunicación como método de aprendizaje. Ante esta posición, deberíamos recordar que las comunidades indígenas mexicanas realizan verdaderos esfuerzos por mantener viva la difusión de las traidiciones y costumbres típicas de la cultura maya. Es lógico entender que los miembros más activos de la comunidad inviertan tiempo en la difusión de leyendas e historias que en métodos de educación alternativos como la educacomunicaicón. Frente al $60 \%$ de estudiantes que no sabía nada sobre la existencia de la educomunicación, hay un porcentaje del $40 \%$ del alumnado que sí ha leído, investigado o accedido a informaciones relacionadas con el desarrollo del método educomunicativo. Un $10 \%$ de los estudiantes encuestados no saben/no contestan la cuestión.

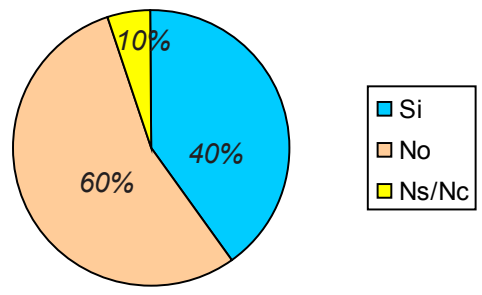

Gráfico 1. Fuente: Elaboración propia 
En este gráfico podemos ver la importancia que tienen los métodos tradicionales de educación frente a métodos innovadores basados en la educomunicación. En el caso de los estudiantes mexicanos, un $50 \%$ de los alumnos prefieren el método de educación tradicional que siguen los maestros de la comunidad de Ucú. Este método se basa en la difusión de tradiciones y cultura del poblado, origen de otras tribus mexicanas y la importancia de mantener vivo el legado de la comunidad. Frente a este porcentaje, un $40 \%$ de los estudiantes considera que el método basado en la educomunicaicón, es decir, el experimento televisivo, ha sido más provechoso y que han aprendido más con esta metodología que con el modelo tradicional. Tan sólo un $10 \%$ de los estudiantes No sabe/no contesta a la cuestión planteada.

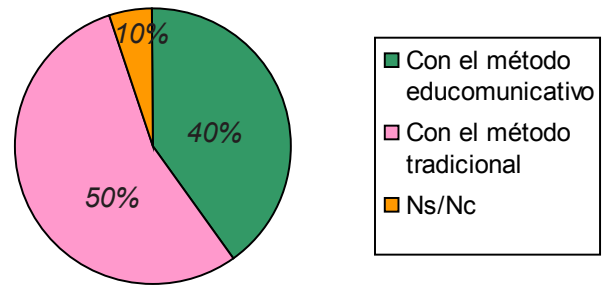

Gráfico 2. Fuente: Elaboración propia.

Por área de interés, los estudiantes de la comunidad mexicana de Ucú valoran el aprendizaje desde el conocimiento práctico (52\%), seguido de otras áreas de interés como son la adquisión de un aprendizaje participativo (45\%) o incluso la capacidad para mejora las relaciones con otros compañeros de clase (15\%). Este gráfico nos ofrece información sobre los múltiples beneficios que tiene la aplicación de la educomunicación en las aulas, como un modelo de educación diferente a los sistemas tradicionales de enseñanza.
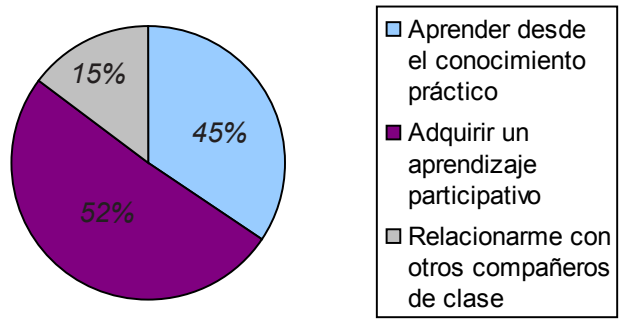

Gráfico 3. Fuente: Elaboración propia.

En este gráfico podemos ver como los estudiantes de la comunidad rural tienen diferentes perspectivas sobre lo qué es la educomunicación. La gran mayoría considera que la educomunicación es un modelo de educación basado en la enseñanza desde los medios de comunicación (no es del todo cierto pero es una de las razones fundamentales por las cuales la educomunicación existe para fomentar la educación desde los medios de comunicación), otro porcentaje analizado considera que la educomunicación es una metodología de estudio práctico (30\% de los encuestados) frente a una franja minoritaria que apunta a la educomunicación como una nueva dinámica de estudio teórico $(25 \%)$. 


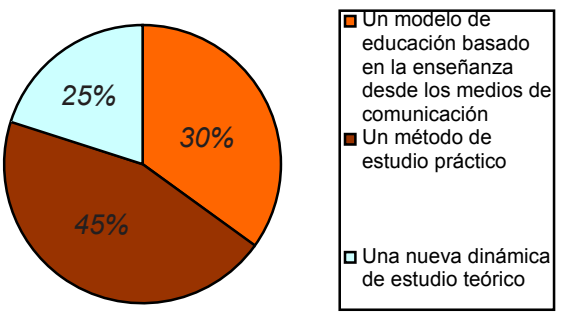

Gráfico 4. Fuente: Elaboración propia.

El último gráfico, en base a las preguntas realizadas a los encuestados, nos ofrece información sobre la importancia que ha tenido la experiencia educomunicativa entre los jóvenes de la comunidad indígena. Un 38\% de los encuestados consideran que con el género televisivo del magazine han aprendido a trabajar en equipo, algo que en un futuro próximo pondrán en práctica en cualquier trabajo que realicen. Un amplio porcentaje de los alumnos considera que con el magazine televisivo ha aprendido a trabajar en equipo, frente a un $27 \%$ que señalan la importancia del experimento educomunicativo como un canal fundamental para mejorar desarrollar áreas personales del conocimiento.
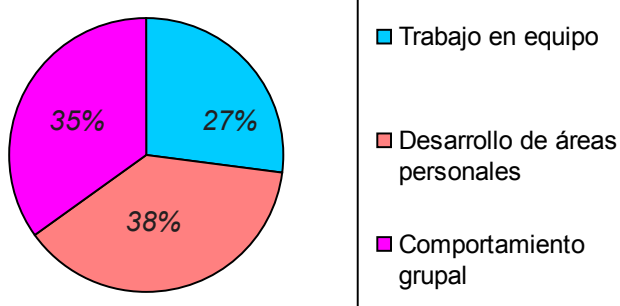

Gráfico 5. Fuente: Elaboración propia.

Este gráfico es uno de los más importantes de todos obtenidos. Responde a la variable "calidad de contenidos en el programa de televisión diseñado frente a los contenidos existentes en la televisión". Como puede verse, un amplio número de estudiantes encuestados, un 50\%, consideran que el formato "Televisión Educativa" es más útil para su formación que el resto de contenidos existentes en la televisión. Así, un $30 \%$ de los encuestados señala que el principal interés de este proyecto ha sido la posibilidad de fomentar la calidad de contenidos, frente a un $20 \%$ de los estudiantes que no dudan en afirmar que el principal atractivo de este proyecto ha sido la creación de un proyecto televisivo acorde a sus necesidades.

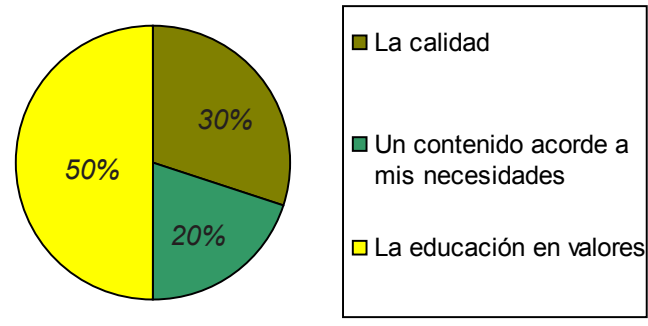

Gráfico 6. Fuente: Elaboración propia. 


\subsection{Resultados de estudiantes españoles}

Este gráfico muestra el absoluto desconocimiento que existe en buena parte de los centros de educación secundaria obligatoria en relación a la existencia de la educomunicación. Un 70\% de los alumnos de ESO del instituto Tirso de Molina de Córdoba no tenían conocimiento de la existencia de la educomunicación como un método real y eficaz para enseñar. Es un porcentaje de estudiantes muy alto. Frente a esta aplastante mayoría, tan solo un $10 \%$ conocen la educomunicación como alternativa a la educación tradicional. Este conocimiento puede deberse a que los padres y madres les hablan de otros métodos en casa. Por último, un $20 \%$ del alumnado no contesta a esta cuestión.

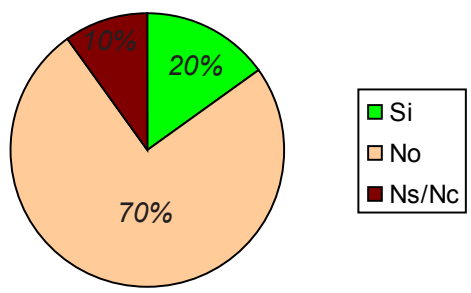

Gráfico 7. Fuente: Elaboración propia.

Sin lugar a dudas, mediante este gráfico podemos comprobar como la aplicación del método educomunicativo en los centros educativos es beneficiosa y necesaria para fomentar el conocimiento entre los estudiantes. Un $70 \%$ de los encuestados considera que ha aprendido más con el método de estudio y enseñanza basado en la educomunicación, frente un $25 \%$ de estudiantes que señalan su preferencia a las formas tradicionales, o un $5 \%$ que no sabe y no contesta a esta pregunta.

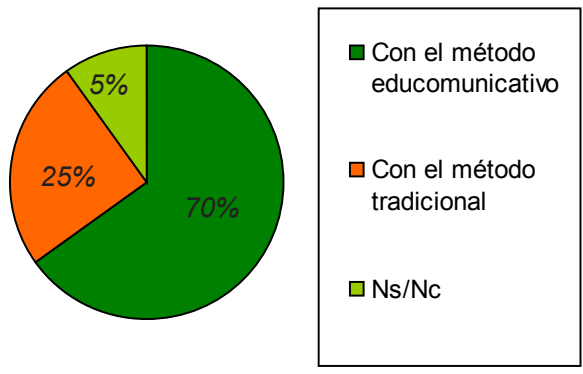

Gráfico 8. Fuente: Elaboración propia.

Para los estudiantes, el principal interés o atractivo de la experiencia educomunicativa ha radicado en la posibilidad de reforzar ciertas áreas de su personalidad que posteriormente pondrán en práctica en cursos superiores, asignaturas del mismo curso en su vida profesional. Un $65 \%$ de los encuestados considera que lo que más interés le ha despertado de la educomunicación ha sido la posibilidad de aprender desde el conocimiento práctico y real, frente a un 35\% que señala la opción de adquirir un aprendizaje participativo, o un $10 \%$ final que apunta como dato de interés la mejora en la relaciones personales con otros compañeros de clase. 


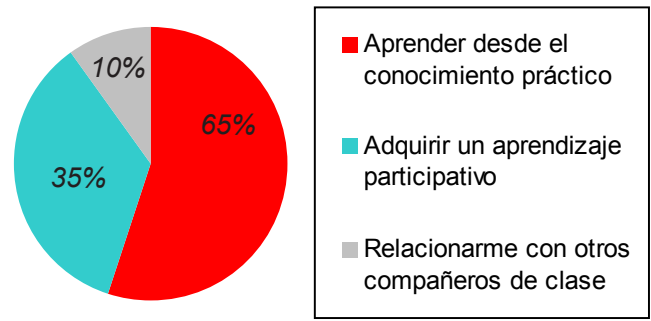

Gráfico 9. Fuente: Elaboración propia.

Una de las cuestiones más importantes de esta investigación ha sido la implementación, explicación y difusión del método educomunicativo entre los alumnos seleccionados. En el caso de los estudiantes cordobeses, un 45\% de los alumnos señalan que la educomunicación es un método de estudio práctico (en realidad es así pero también tiene conexiones con diferentes teorías y con otras disciplinas del conocimiento). Un porcentaje amplio, un 35\%, señala que la educomunicación es una nueva dinámica de estudio, frente a un $20 \%$ minoritario que considera la educomunicación como una nueva dinámica de estudio teórico.

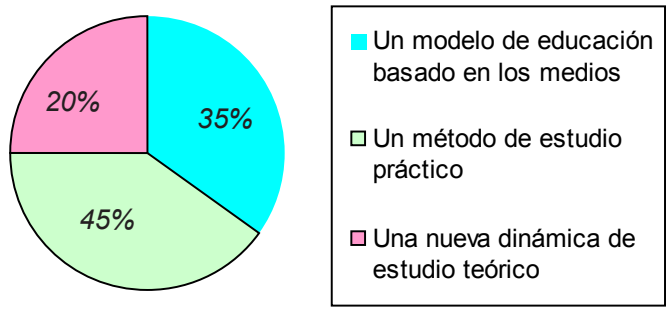

Gráfico 10. Fuente: Elaboración propia.

El gráfico más importante de todos los analizados hasta ahora. Tanto para los estudiantes mexicanos como para los estudiantes españoles, lo más importante de la experiencia educomunicativa es la posibilidad de aprender a trabajar en equipo $(35 \%)$, la enseñanza y reforzamiento del comportamiento grupal $(38 \%)$ y el desarrollo de áreas personales (27\%).

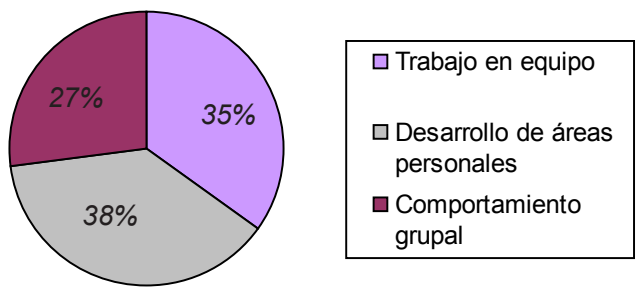

Gráfico 11. Fuente: Elaboración propia.

Al igual que en el caso de los estudiantes mexicanos, este gráfico muestra el principal interés que ha tenido para los estudiantes españoles la actividad educomunicativa. La elaboración de un programa de televisión bajo criterios de calidad mediática ha despertado el interés principal de los estudiantes cordobeses. Los alumnos han comprobado cómo es posible generar información de calidad, acorde a los parámetros de calidad periodística. Por otra parte, un $35 \%$ de los estudiantes señala la importancia de la actividad educomunicativa como un instrumento que refuerza la educación en valores. Finalmente, un minoritario porcentaje del $20 \%$ considera que el principal 
atractivo del proyecto "Televisión Educativa" ha sido la visibilidad de un contenido acorde a los valores y preocupaciones actuales de la comunidad estudiantil.
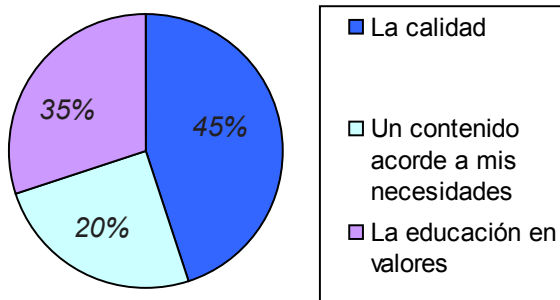

Gráfico 12. Fuente: Elaboración propia.

\section{Conclusiones}

La educomunicación, como fénomeno aglutinador de la educación y la comunicación, deja tras de sí importantes reflexiones. La dinámica educomunicativa potencia la creación del espíritu crítico entre hombres y mujeres, fomenta la convivencia en el entorno y consolida los saberes tradicionales con un pie en el futuro. La experiencia educomunicativa en América Latina y en España ha confirmado que existe otro método de enseñanza. Comunidades rurales, grandes urbes, aldeas, ciudades...en cualquier asentamiento geográfico la educomunicación registra un crecimiento en alza.

La experiencia educomunicativa realizada en el asentamiento rural de Ucú y en el instituto cordobés Tirso de Molina genera datos concluyentes. El esfuerzo conjunto de profesores, alumnos, estudiantes y miembros tribales demuestra que otro modelo de educación desde la comunicación es posible. La creación de contenidos en el programa "Televisión Educativa" respondía a las demandas de los niños (contenidos de salud, alimentación, ocio, cultura), pero también ha servido para que los chicos y chicas participantes de este proyecto puedan desarrollar otras áreas de su personalidad y por supuesto, les ha servido para mejorar y desarrollar aspectos que un futuro próximo tendrá que aplicar en cualquier trabajo como son las capacidades para trabajar en equipo o la admisión en una organización grupal.

Las hipótesis han sido confirmadas. Por una parte, mediante los datos obtenidos, se ha constatado la hipótesis que afirmaba que la experiencia educomunicativa sirve para que los estudiantes aprendan valores que posteriormente pondrán en práctica en cursos superiores o en el futuro profesional. Los resultados obtenidos, tanto en el caso del alumnado mexicano como el alumnado español, confirma que los estudiantes necesitan aprender contenidos teóricos, sí, pero también tienen que adquirir las claves que les ayuden a tener un futuro digno y brillante, tal y como ellos merecen. La segunda hipótesis a confirmar consideraba la educomunicación como un modelo de contenidos en el que el pluralismo, la libertad y la educación en valores esta asegurada frente a la distorsión de información del discurso televisivo actual imperante en cadenas de televisión. Esta hipótesis también se ha confirmado. Los estudiantes tuvieron la oportunidad de elaborar su propio contenido, acorde a sus necesidades e inquietudes, difundiendo noticias que tienen que ver con sus preocupaciones cotidianas presentes y futuras. 


\section{Referencias bibliográficas}

Aparici, R. (coord.) (2010a). Educomunicación: más allá del 2.0. Gedisa, Barcelona.

Aparici, R. (coord.) (2010b). Conectados en el ciberespacio. Madrid: UNED.

Beltrán, L. R. (2005). La comunicación para el desarrollo en Latinoamérica. Un recuento de medio siglo. III Congreso Panamericano de la Comunicación. Buenos Aires (Argentina). Recuperado de www.infoamerica.org/teoria_textos/lrb_com_desarrollo.pdf.

Bohm, D. (1997). Sobre el diálogo. Barcelona: Kairós.

Bohm, D. y Peat, D. (1988). Ciencia, orden y creatividad. Barcelona: Kairós.

Bourdieu, P. y Passeron, J. (2001). La reproducción. Elementos para una teoría del sistema de enseñanza. Madrid: Editorial Popular.

Castells, M. (2005). La era de la información. Vol. I. La sociedad red. Madrid: Alianza.

Chomsky, N. y Ramonet, I. (2008). Cómo nos venden la moto. Información, poder y concentración de medios. Barcelona: Icaria.

Correa, R. I. (2001). La sociedad mesmerizada. Medios, nuevas tecnologías y conciencia crítica en educación. Universidad de Huelva.

Correa, R. I. (2002). El hilo de Ariadna. Revisión crítica de los contextos educativos de la sociedad neoliberal. Huelva: Grupo Ágora. Recuperado de http://www.uhu.es/ramon. correa/medios_com_social/documentos/docs/libros_libres/hilo_ariadna.pdf.

De Oliveira, I. (2000). La Comunicación Educación como nuevo campo del conocimiento y el perfil de su profesional. En Comunicación-Educación, coordenadas abordajes, travesías. Bogotá: Universidad Central (27-48).

De Oliveira, I. (2002). Gestión comunicativa y educación: caminos de educomunicación. Comunicación y Educación, ECA/USP. Sao Paulo: Editora Segmento.

Deleuze, G. y Guattari, F. (1997). Rizoma (Introducción). Valencia: Pre-textos.

Díaz Bordenave, J. y Martíns De Carvalho, H. (1978). Planificación y comunicación. Quito, Ecuador.

Flecha, R.; Davila, A. y Vargas, J. (2004). Metodología comunicativa crítica en la investigación en ciencias sociales: la Investigación Workaló. Lan Harremanak: Revista de Relaciones Laborales, № 11, 21-34. Universidad del País Vasco.

Freire, P. (1967). La educación como práctica de la libertad. Montevideo: Tierra Nueva.

Freire, P. (2005). Pedagogía del oprimido. México: Siglo XXI.

Gumucio-Dagron, A. \& Tufte, T. (eds.) (2006). Communication for social change anthology: historical and contemporary readings. New Jersey: Communication Social Change Consortium.

Gutiérrez, A. (2003). Alfabetización digital. Algo más que ratones y teclas. Barcelona: Gedisa.

Hernández Huerta, J. L. (2012). Freinet en España (1926-1939). Escuela popular, historia y pedagogía. Valladolid: Castilla Ediciones.

Huergo, J. (2003). El reconocimiento del "universo vocabular" y la prealimentación de las acciones estratégicas. La Plata: Centro de Comunicación/Educación y Cátedra 
de Extensión Agropecuaria (UNPL) (2005). Hacia una genealogía de Comunicación/ Educación. Rastreo de algunos anclajes político-culturales. La Plata: Ediciones de Periodismo y Comunicación (UNLP).

Kaplún, M. (1997). De medios y fines en comunicación. Revista Chasqui, n 58. Recuperado de http://chasqui.comunica.org/kaplun.htm.

Kaplún, M. (1998). Una pedagogía de la comunicación. Ediciones de la Torre, Madrid.

Levy, P. (2004). Inteligencia colectiva. Por una antropología del ciberespacio. Organización Panamericana de la Salud. Recuperado de http://inteligenciacolectiva.bvsalud. org/public/documents/pdf/es/inteligenciaColectiva.pdf.

Mata (2010). Didáctica de la Educomunicación. Recuperado de http://didcticadelaeducom.wordpress.com/educomunicacion

Martín Barbero, J. (2001). El libro y los medios, crítica de la razón dualista. Educación desde la Comunicación. Madrid: Editorial Norma.

Masterman, L. (1983). La educación en materia de comunicación: problemas teóricos y posibilidades concretas. Perspectivas, vol. XIII, n² 2, 191-200. París: Unesco.

Masterman, L. (1993). La enseñanza de los medios de comunicación. Madrid: Ediciones de la Torre.

Macbride, S. (dir.) (1980). Un solo mundo, voces múltiples. Comunicación e información en nuestro tiempo. México: Fondo de cultura económica. Recuperado de http://unesdoc.unesco.org/images/0004/000400/040066sb.pdf

Pasquali, A. (1963). Comunicación y cultura de masas. Comunicar, $n^{\circ} 29, \mathrm{XV}, 2007$, España.

Sánchez Bravo, A. (1978). Objetividad en el discurso informativo. Madrid: Ediciones Pirámide.

Singhal, A. (2008). Cultura popular con un propósito: Uso de medios de eduentretenimiento para el cambio social. Recuperado de http://www.comminit.com/es/node/307184.

Valderrama, Carlos Eduardo (2000). Introducción en Comunicación-Educación, coordenadas abordajes, travesías. Universidad Central. 
